\title{
El Brazo Robótico como herramienta pedagógica en el aula de clase
}

\section{The robotic arm as a teaching tool in the classroom}

Ingrid Johana Henao', Jorge Armando Giraldo', Ferney Alexander Meza', Carlos William Sánchez ${ }^{2}$ y John Edward Ordoñez ${ }^{2}$

1 Fundación Universitaria Católica Lumen Gentium, Programa de Ingeniería Industrial, Facultad de Ingeniería, Cali, Valle del Cauca-Colombia.

${ }^{2}$ Fundación Universitaria Católica Lumen Gentium, Grupo de Investigación KIMSA, Departamento de Ciencias Básicas, Facultad de Ingeniería, Cali, Valle del Cauca-Colombia.

Autor para correspondencia:

csanchez@unicatolica.edu.co

\section{Resumen}

En este artículo se explica en qué consiste el desarrollo del proyecto Brazo Robótico. Un sistema de componente tecnológico, para la formación de los futuros ingenieros industriales de UNICATÓLICA. El desarrollo de esta interfaz se hará, principalmente, en el marco de la filosofía del software libre. Con la construcción e implementación del brazo robótico, los estudiantes podrán realizar actividades, como transporte de objetos o selección de piezas defectuosas en una línea de producción; entre otras. Se trata de una solución de carácter tecnológico que, además de innovar, contribuye a los procesos de enseñanza y es un motor motivacional para los estudiantes en el aula de clase. En el proceso se desarrollarán circuitos de control de los motores que le darán los diferentes grados de libertad al sistema. Para esto, se usará la plataforma de hardware libre Arduino. El resultado de esta implementación se verá reflejado en los egresados, quienes con esta formación, podrán destacarse en el ámbito laboral, tan competitivo actualmente.

Palabras clave: robot, antropomórfico, automatización, brazo robótico.

\begin{abstract}
In this paper, the development of the Robotic Arm project is analyzed. A system of technological component, for the education of the future industrial engineers of UNICATOLICA. The development of this interface will be elaborated mainly within framework free software philosophy. With the construction and implementation of the robotic arm, students will be able to carry out activities, such as transportation of objects, selection of defective parts in a production
\end{abstract}


line, among other. It is a technological solution that, in addition to innovating, contributes to the teaching processes and is a motivational motor for students in the classroom. In the process, engine control circuits will be developed, for the different degrees of freedom of the system. For this proposal, Arduino platform based on free hardware will be used. The result of this implementation will be reflected in the student graduates, who, with this education, will be able to stand out in the labor field, so competitive at the moment.

Keywords: robot, anthropomorphous, automation, robotic arm.

\section{Introducción}

El uso de tecnologías novedosas en el aula de clase, se plantea como una herramienta para la enseñanza, con la cual se podrán desarrollar nuevas competencias y mejorar el proceso de apropiación del conocimiento en los estudiantes. La fabricación de un brazo robótico y su implementación, como herramienta didáctica en el aula de clase, es una alternativa a la simulación de los procesos técnicos, que son la herramienta disponible. Sin embargo, dicha simulación no ofrece el elemento de interactividad necesario para estimular el proceso de aprendizaje.

Hoy en día, se escucha con frecuencia, entre los profesores de los departamentos de investigaciones de las instituciones de enseñanza, preguntar por las condiciones y requisitos para implementar proyectos educativos que incluyan la robótica, como herramienta, en el proceso de enseñanza en el aula de clase. La indagación sobre estas necesidades reveló que, en la mayoría de los casos, el interés surge por el impulso que marcan los avances tecnológicos, presentes en el día a día. Por ejemplo, los sistemas autónomos rodean una persona desde el inicio de su jornada, en un día cualquiera. Estos pequeños robots gobiernan el entorno, en forma imperceptible y generan una atmósfera de bienestar que se disfruta inconscientemente. Muestra de ellos son los celulares. Estas máquinas autónomas detectan, incluso, la mirada del usuario, su dedo, su posición geográfica, la intensidad de la luz, etc. Otros casos son la lavadora, con ciclos programables; la nevera, con ciclos de frío dirigidos y algunas hasta con capacidad de conectividad; televisores que no solo proyectan una imagen, sino que ofrecen enlace de datos vía Internet; los relojes modernos que monitorean la presión arterial del que los porta y muchos más. Variados ejemplos se pueden encontrar en la obra de Hakima, C. (2010), quien ilustra la forma en que estos dispositivos se conectan entre sí.

Dentro del ámbito académico, es posible encontrar robots computacionales que facilitan la interacción entre los estudiantes y la institución. Por ejemplo, la implementación de una plataforma virtual que permite al estudiante conocer todo el contenido del curso, descargar material de apoyo, o comunicarse con el profesor de la asignatura, empleando simplemente un ordenador, o teléfono móvil. Tal es el caso de la plataforma Moodle, de la cual Valenzuela y Pérez (2013) hacen un análisis acerca de la contribución a la formación académica de este tipo de aplicaciones gobernadas por robots informáticos. Otros sistemas autónomos informáticos se establecen para suplir la necesidad de socialización humana. Como las redes sociales que no solo comparten fotos, sino videos. De esta manera, fortalecen las relaciones estudiante-estudiante, estudiante-docente, estudiante-universidad y universidad-sociedad, en general. Estos sistemas automatizados son conocidos como"robots web", o"web crawlers". En su tesis doctoral,Yang Sun (2008) hace un análisis exhaustivo de estos sistemas. El desempeño de estos aparatos ha sido tan eficiente que ha generado estados de bienestar y complacencia, manifestados en comportamientos adictivos hacia este tipo de tecnologías. Haythornthwaite, C. (2005) hace un estudio sobre esto.

Todo esto conduce a cuestionarse ¿Es posible establecer una línea de investigación en automatización y robótica en UNICATÓLICA?, ¿cómo establecer desarrollos que permitan construir herramientas didácticas de apoyo en el aula de clase, con el componente tecnológico como protagonista?

Garnica E. (2013) explica cómo el uso de herramientas automatizadas, desde una perspectiva didáctica, constituye un elemento de fácil inclusión en el aula de clase. Todo, porque, para el estudiante, es un proceso casi natural experimentar con interfaces hombre-máquina, desde muy corta edad y con diferentes 
automatismos. Mirado desde esta perspectiva, esta es una oportunidad para aprender acerca de estos sistemas, de su interacción con el estudiante en el aula de clase y de las posibles aplicaciones que la interacción estudiante-docente genere en diferentes niveles. Por supuesto, acompañándolo con el aprendizaje de competencias, porque la meta es que los estudiantes sean profesionales capacitados en dichas tecnologías.

Antes de entrar en materia, es conveniente definir algunos conceptos. Robot Institute of America (1979) dice que el robot industrial es "Un manipulador multifuncional reprogramable, capaz de manipular y/o mover piezas, herramientas o dispositivos especiales, según trayectorias variadas, programadas para realizar diferentes trabajos". Obviamente, se trataba de una definición muy limitada para lo que hoy constituyen estos sistemas. El diccionario Webster (2009) lo define como: “Un dispositivo automático que realiza funciones normalmente atribuidas a los seres humanos o una máquina en la forma de un ser humano". Este concepto es mucho más amplio, pues tiene en cuenta los avances relacionados con la Inteligencia Artificial que hacen parte de los sistemas robóticos modernos.

Los procesos industriales actuales requieren sistemas que ejecuten tareas específicas con suficiente flexibilidad, precisión y/o fuerza; para que puedan manipular cualquier tipo de objetos, indistintamente de su tamaño, peso y forma. Por este motivo, se han venido incorporando brazos robóticos, como elementos terminales de manipuladores robóticos en los procesos industriales. Las empresas necesitaban realizar tareas más precisas y con una mayor destreza, que emularán las funciones de la mano humana. Estas máquinas, por sus características, son capaces de sujetar y manipular objetos con formas y tamaños diferentes, lo cual proporciona, además, la posibilidad de ejecutar movimientos de fuerza y precisión, estables y con gran destreza.

\section{La robótica como herramienta pedagógica en el aula de clase}

En los trabajos de Garnica E. (2013), Jiménez, L. (2013), Márquez D. et al (2014), Lancheros, D. y Ru- biano, A. (2009), Lancheros, D. (2010) y Patiño, K. et al (2010), se habla de la robótica pedagógica como una disciplina que tiene por objeto la generación de ambientes de aprendizaje, basados, fundamentalmente, en la actividad de los estudiantes. Por ejemplo, aplicar métodos abordados en la teoría, para facilitar el aprendizaje, el desarrollo y la práctica de diferentes proyectos en la solución de problemas industriales. En efecto, se está llevando a cabo un acercamiento para la solución de problemas derivados de distintas áreas del conocimiento, como las matemáticas, las ciencias naturales y experimentales, la tecnología y las ciencias de la información y la comunicación; entre otras. Mediante estos procesos, se generan ambientes de aprendizaje innovadores, en los que se permite a los estudiantes simular fenómenos y mecanismos, diseñar y construir prototipos de sistemas productivos que los llevan a proponer soluciones aproximadas a la realidad.

Gracias a la naturaleza multidisciplinaria de la robótica, es posible involucrar una gran cantidad de áreas del conocimiento, tales como matemáticas, física, electrónica, computación, visión e inteligencia artificial; entre otras. Es importante mencionar que, a pesar de que la robótica es un área eminentemente experimental, todos sus resultados están sustentados con un estricto rigor científico. Por esto, la robótica educativa es un ente, en el cual concurren muchos elementos del conocimiento. Además, convergen conceptos transmitidos por los docentes, en un contexto de aprendizaje apoyado en las tecnologías digitales. De esta manera, se hace robótica y se involucra -a quienes participan- en el diseño y construcción de creaciones propias (objetos que poseen cuerpo, control y movimientos); primero, mentales y luego, físicas; construidas con diferentes materiales y controladas por un computador; son las llamadas simulaciones o prototipos. Estas creaciones pueden tener su origen, en un referente real.

\section{La evolución de la pedagogía en el aula de clase}

Muchos docentes nos preguntamos: ¿Es necesaria la evolución de la pedagogía en el aula de clase?, ¿por qué es necesario introducir nuevas herramientas didácticas en el aula de clase? 
Dobzhansky et al. (1993), definen la evolución como el proceso por el cual una especie sufre cambios aleatorios. Explican que si son benéficos en la adaptación, constituyen una mejora para la especie, y la convierten en una nueva. Este proceso suele darse de manera muy lenta, pues deben transcurrir muchas generaciones antes de que comience a hacerse evidente alguna variación. Darwing (1859) planteó, en su famosa publicación. El Origen de la Especies, que estas evolucionan para lograr su adaptación a nuevos entornos. Aunque es una percepción un poco determinista para un evento que hoy se sabe es aleatorio; ideológicamente, infiere más el concepto de adaptabilidad, pues busca sacar un mejor provecho del entorno. Partiendo de esta premisa y aplicándola al entorno educativo, es válido plantear la necesidad de evolucionar los métodos de enseñanza e insertar herramientas tecnológicas para mejorar el proceso de apropiación del conocimiento. Esto, a la vez que motiva y facilita el aprendizaje en los estudiantes, contribuye a la adaptación a las nuevas disciplinas vigentes.

Autores -como Bayraktar, S. (2000-2001), Brown, J. (1993), Daniel (2012), David (2012) y Watson, D. (2001) - se muestran cautos a la hora de recomendar la inserción de las TIC, en el aula de clase. Ellos exponen casos documentados, en los que esta introducción desmedida puede desencadenar un proceso de desconexión del estudiante con los conceptos y con el proceso mismo de formación. Los métodos tradicionales, comprobados ampliamente, permiten impartir el conocimiento, manteniendo el contacto profesor-alumno; tan importante, no solo para fortalecer la relación, sino para transferir conceptos, experiencia y valores.

Otros autores, como Seidel (1994) y Hatch (1997), son más optimistas en la inclusión de estas tecnologías, en la academia. Aunque la mayoría de estos trabajos se centran en la computadora, como elemento de inclusión tecnológica, ha habido una diversificación, en los últimos años, con la aparición de las tabletas digitales, teléfonos inteligentes, aulas inteligentes; entre otros. La inserción de complementos robóticos -en el aula de clase, para incentivar el desarrollo de nuevas competencias- es tan solo la evolución de las ideas gestadas anteriormente. El estudiante debe usar continuamente estas tecnologías, para estar a la par del cambiante conocimiento alrededor de los principios científicos y la forma como se accede a ellos. Es la única manera como el estudiante podrá desarrollar dichas habilidades.

Las percepciones de los autores mencionados dependen fuertemente de la escuela de pensamiento a la que pertenecen, como también de su experiencia en los procesos de formación; dado que ambos tienen fundamentos que constituyen posturas diferentes. Sin embargo, no se puede desconocer la base de sus investigaciones. Hay que tenerlas en cuenta a la hora de implementar estas tecnologías en el aula de clase, para no caer en procesos de desinformación.

\section{La motivación de los estudiantes}

Es preciso cuestionarse: ¿Cómo motivar a aquellos estudiantes que necesitan un poco más que los cursos? En todas las aulas de clase, siempre uno o varios estudiantes preguntan: ¿Cuál es la utilidad de aprender Física, Cálculo, Algebra y Trigonometría? Estos son los que no están motivados a resolver las derivadas o integrales, los complejos ejercicios de las leyes de Newton o el escalonamiento de aquellos sistemas de ecuaciones, que los docentes suelen dejar como tarea; tampoco están en la capacidad de visualizar cómo el arduo trabajo realizado puede conducirlos a un resultado tangible.

Un caso representativo -en el que el estudiante siente que su esfuerzo va más allá de un resultado numérico- es el simple montaje de un circuito en serie y paralelo. Con la ayuda de una batería y unas resistencias eléctricas, podrá encender las bombillas sin riesgo de quemarse; previamente, tiene que haber hecho unos cálculos sencillos que describen estos fenómenos (Ley de Ohm y Leyes de Kirchoff). Entonces, hasta el más desentendido de la clase comienza a asociar los conceptos simples en desarrollos electrónicos de la vida cotidiana y pensando en potenciales aplicaciones.

¿Qué sintió Tomas Alva Edison cuando logró producir su primer bombillo?, ¿cuál fue la impresión de Henry Ford, al ver en marcha su primer vehículo? Es común que, en estos casos, los estudiantes demanden algún espacio físico y organizado, dentro de la institución, donde puedan despejar las dudas suscitadas en las prácticas sencillas de laboratorio de física. $\mathrm{O}$, tam- 
bién, poder revisar el Internet, para replicar algunos de los desarrollos allí descritos; pero teniendo una absoluta comprensión de los conceptos básicos que se esconden detrás de ellos.

En este caso, UNICATÓLICA puede ofrecer alternativas para que el estudiante, mediante el ejercicio de la práctica, aprenda conceptos, los formalice e interiorice.Y, a la vez, adquiera habilidades en el planteamiento y la solución de problemas.

\section{Implementación de este tipo de alternativas tecnológicas en UNICATÓLICA}

El desarrollo de tecnologías de bajo costo, como la plataforma Arduino, es posible en UNICATÓLICA. Esta es una tarjeta electrónica desarrollada en Italia en el 2005. En sí, es un dispositivo programable, con una base de tiempo, con puertos de entrada y salida de información, el cual puede usarse para procesos de automatización. Ejemplos de esto se pueden consultar en los trabajos de Banzi (2014), Margolis (2011) y Simon (2011). En efecto, es un sistema autónomo que no necesita otro elemento para ser configurado, porque es un instrumento de fácil configuración y aplicación. El desarrollo, por terceros, de módulos -ciento por ciento compatibles- brinda una oportunidad histórica para la institución y, en general, para cualquier universidad que requiera implementar este tipo de tecnologías en el aula de clase. Su naturaleza modular y escalable permite que los estudiantes, con poco fundamento en el área de la electrónica, se aventuren en el desarrollo de proyectos robustos, con la certeza de un entendimiento profundo de los elementos involucrados.

Basados en todas las premisas anteriores, se propone desarrollar un brazo robótico, como herramienta para la enseñanza y profundización en el diseño, análisis y mejoras de sistemas productivos. Así, es posible comparar la oferta tecnológica de UNICATÓLICA con la que ofrecen otras universidades de la región, las cuales cuentan con este tipo de máquinas y módulos, para capacitar a sus estudiantes. En UNICATÓLICA, los efectos serán evidentes: se forjarán mayores oportunidades educativas y laborales que generan valor agregado en la industria; se mejorarán las competencias de los estudiantes, lo cual permitirá, a nuestros egresados, insertarse laboralmente con habilidades, potencialidades y visión progresista de los procesos productivos.

El proyecto de desarrollo de un brazo robótico, con fines pedagógicos, facilita la enseñanza y adquisición de habilidades en los estudiantes. Esto los conducirá a proponer soluciones a problemas relacionados con los métodos de manufactura avanzada, e incursionarán en el campo de la automatización y de la robótica. Para esto, una vez finalizada la fabricación del brazo, se procederá con la implementación de una serie de prácticas de laboratorio, destinadas a que los estudiantes adquieran habilidades en el manejo de este tipo de tecnologías. En la actualidad, los procesos industriales son de gran importancia y cuentan con este tipo de ayudas tecnológicas, lo cual permite a los sectores productivos ahorrar tiempo y dinero.

Con el fortalecimiento del Grupo de Investigación KIMSA y la aprobación del proyecto de investigación interno "Plataforma móvil para monitoreo y control de variables en ambientes contaminantes o peligrosos de difícil acceso", se fortalece el área de investigación y desarrollo de este tipo de tecnologías. De hecho, servirá como apoyo en el proceso de enseñanza de las asignaturas asociadas con los procesos industriales, tales como diseño de sistemas productivos. Con este trabajo, el programa de Ingeniería Industrial, de la Fundación Universitaria Católica Lumen Gentium, tiene la posibilidad de actualizar sus métodos de enseñanza, pues podrá ofrecer los mismos avances tecnológicos en estas competencias, que las demás universidades de la región.

Al revisar el campo regional, se encuentra que universidades del departamento -como la Autónoma de Occidente, la Valle, la ICESI y el SENA; entre otras- cuentan con este tipo de herramientas tecnológicas en el campo de la robótica. De esta manera, analizan los procesos industriales, desde el punto de vista de la automatización y optimización. A continuación, se describen algunos de los entes educativos y sus proyectos en materia de investigación:

La Universidad Autónoma de Occidente tiene varios grupos de investigación que trabajan en el área. Uno de ellos es el Grupo de Investigación en In- 
geniería Biomédica (GBIO), con categoría A de COLCIENCIAS. Este grupo tiene varios proyectos en curso, entre los cuales se pueden destacar: "Diseño y construcción de una plataforma portátil para la medición de fuerza en estudios de biomecánica deportiva", "Diseño de un sistema de asistencia híbrida para bipedestación y marcha en personas con lesión de la médula espinal", "Desarrollo de estrategias que fomenten una cultura investigativa en los estudiantes de pregrado de Ingeniería Biomédica de la Universidad Autónoma de Occidente". El Grupo de Investigación en Materiales Avanzados para Micro y Nanotecnología (IMAMNT) adelanta proyectos como: "Diseño de un Brazo Robótico para la Automatización del Proceso de Tratamiento de Materiales Sol Gel (Dip Coating): JOSI 2.0", “Diseño e Implementación de Robot Paralelo para Posicionar Muestras de Estudio en Plano Vertical". Adicionalmente, el programa de Ingeniería Mecatrónica desarrolló los proyectos “Rediseño de Brazo Robótico para la Automatización de la Tecnología de Tratamiento de Materiales Sol Gel (Dip Coating)".

En la Universidad del Valle, el Grupo de Investigación en Control Industrial (GICI), con Categoría B, COLCIENCIAS, cuenta, en sus espacios, con un robot industrial tipo SCARA de cuatro grados de libertad, con su unidad de potencia y control, con todo el equipamiento para la programación y ejecución de tareas y trayectorias. Además, disponen de una mesa giratoria de dos grados de libertad con la electrónica correspondiente para programar tareas de taller o procesos de empacado. Se usa como dispositivo cooperativo para robots industriales.

La Universidad Icesi, en el 2014, inauguró el Laboratorio de Ingeniería Industrial, el cual cuenta con un brazo robótico que permite que los estudiantes aprendan a automatizar procesos. Lo están haciendo, por medio de simulaciones y operación real del brazo robótico, en la estación estudiantil adquirida para tal fin. El laboratorio de Ingeniería Industrial tiene cuatro espacios para el desarrollo de prácticas de los estudiantes de la Facultad de Ingeniería: Automatización Industrial, Buenas Prácticas Procesos de Alimentos, Prácticas Interactivas y Logística de Almacenamiento.

En definitiva, el desarrollo de este proyecto constituye un factor importante para mejorar la compe- titividad académica y pedagógica de la institución. De hecho, ofrece un sistema de componente tecnológico para la formación de los futuros ingenieros industriales de UNICATÓLICA.

\section{Primer prototipo robótico de UNICATÓLICA}

Para el desarrollo del prototipo, se implementarán cinco fases que permitirán ajustar pormenores inherentes al diseño y a la operatividad del mismo (Figura 1). Primero, se realizará el levantamiento de los requerimientos; seguidamente, se elaborará el diseño funcional; posteriormente, se construirá el brazo y, por último, se realizarán las pruebas de campo.

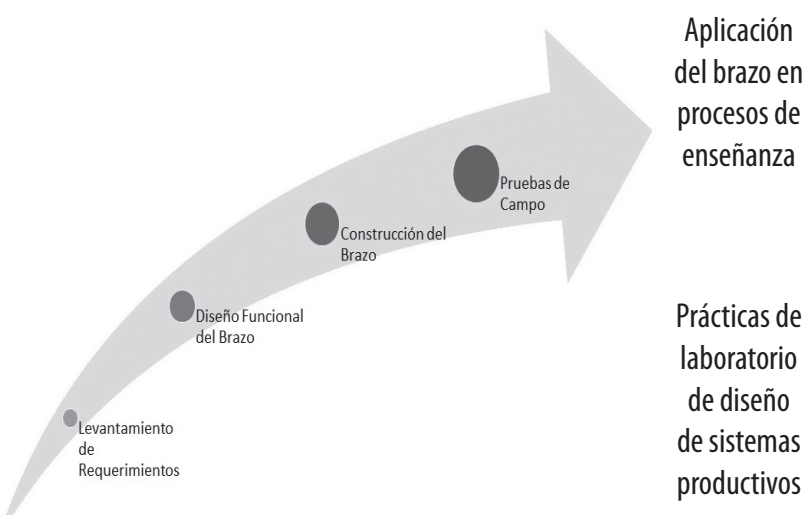

Figura 1. Diagrama de fases en las que se levantará el proyecto. Fuente: elaboración propia

Para realizar el brazo, se utilizará un software de control del mismo, de manera que se pueda alimentar con la información de las trayectorias en el espacio en el cual el brazo debe posicionarse. Esta interfaz se ejecutará, principalmente, con base en la filosofía del software libre.

Otro elemento fundamental en el proceso, es el desarrollo de los circuitos de control de los motores que le darán los diferentes grados de libertad al sistema. Para esto, se piensa usar la plataforma de hardware libre Arduino.

Todo lo anterior, para dar vida al robot antropomórfico de 5 grados de libertad, el cual facilitará la manipulación de diferentes objetos. Una imagen tridimensional del brazo puede observarse en la Figura 2. 


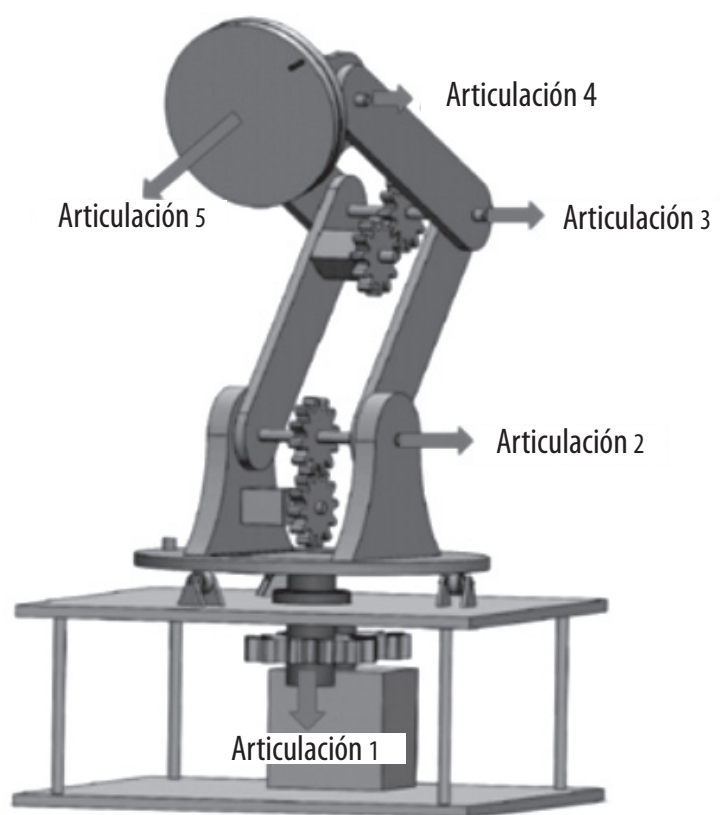

Figura 2. Diseño tridimensional del brazo robótico. Fuente: elaboración propia

El modelo de movimiento del brazo, pensado para este tipo de prototipo, es la parametrización de Denavit-Hartenberg (1955), con el fin de concluir la posición de los ejes de cada una de las articulaciones y sus respectivas orientaciones.

Con la construcción e implementación del brazo robótico, los estudiantes de UNICATÓLICA podrán realizar actividades, como transporte de objetos o selección de piezas defectuosas en una línea de producción; entre otras. Todo esto enfocado en los procesos de enseñanza, para el diseño de sistemas productivos (Figura 3).

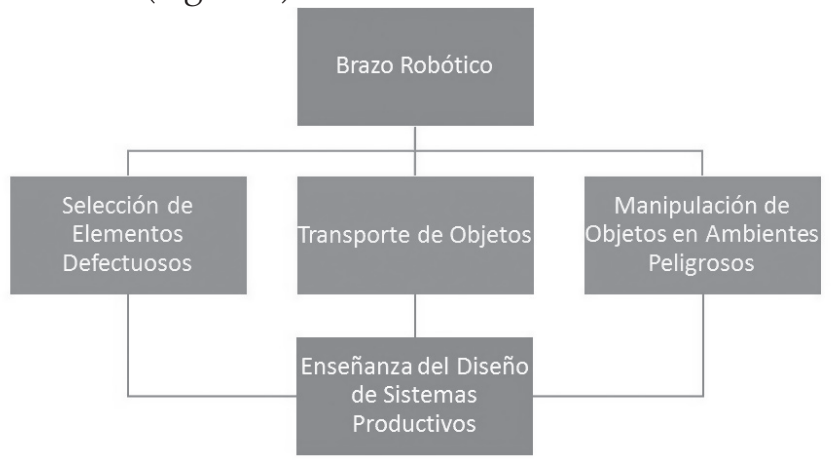

Figura 3. Implementación del brazo robótico en el aula de clase. Fuente: elaboración propia

También, como una herramienta que permita al estudiante la inserción de nuevas tecnologías en el aula de clase. Lo anterior exige la implementación de prácticas de laboratorio, en las que haya una clara interacción entre el brazo robótico y el estudiante. Se debe plantear una serie de lógicas participativas que le permitan adquirir las potencialidades que necesita. Un ejemplo de práctica de laboratorio concebida como punto de partida es el siguiente:

Inserción del brazo en un proceso productivo, para seleccionar el material que se desplaza a lo largo de una banda transportadora (Figura 4). El operario 6 de un proceso que produce 4 tipos de productos debe realizar la operación número 6; adicionalmente, transportar el producto terminado al depósito que corresponde, según el tipo de producto. Como mejora del proceso, el brazo será un seleccionador para eliminar los transportes y fatigas del operario 6 .
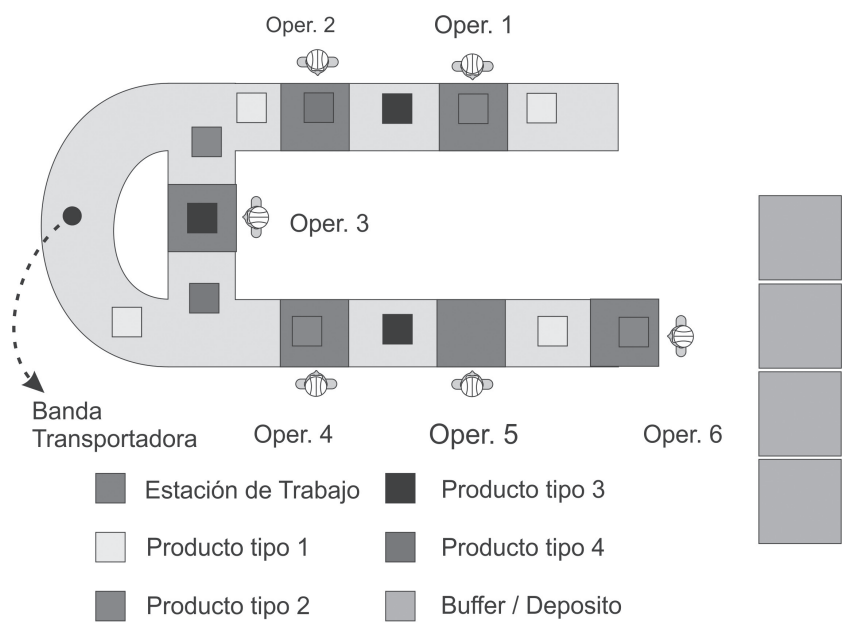

Figura 4. Diagrama de la práctica de laboratorio del brazo robótico.

Fuente: elaboración propia

\section{Conclusiones}

Gracias al estudio explicado, fue posible:

- Identificar la problemática de la inserción de las tecnologías en el aula de clase, partiendo de la necesidad de actualizar los procesos de enseñanza en la Facultad de Ingeniería de UNICATÓLICA, con una perspectiva originada desde el Departamento de Ciencias Básicas.

- Proponer una solución de carácter tecnológico que, además de innovar, contribuye a los procesos de enseñanza y es un motor motivacional para los estudiantes en el aula de clase. 
- Plantear la alternativa de implementar una herramienta tecnológica en las asignaturas, cuyo objeto es el estudio de métodos y tiempos y/o procesos de manufactura avanzada. Por ejemplo, "Diseño de Sistemas Productivos". Esto permitirá que el estudiante interactúe con nuevas tecnologías que hasta el momento no se han trabajado en UNICATÓLICA.

\section{Agradecimientos}

Los autores de este artículo agradecemos a Efraín Ávila, por su invaluable soporte tecnológico al proyecto al que ha aportado ideas y desarrollos propios para hacerla viable.

\section{Referencias}

Banzi, M. (2014). Make: Getting Started with Arduino: The Open Source Electronics Prototyping Platform. Maker Media, Inc. (Eds.). Sebastopol, CA, USA. 262 p.http://www.esc19. net/cms/lib011/TX01933775/Centricity/Domain/110/make_gettingstartedwitharduino_3rdedition.pdf.

Bayraktar, S. (2000-2001). A Meta-analysis of the Effectiveness of Computer-Assisted Instruction in Science Education. J Res Tech Educ, 34 (2), 173-188. http://dx.doi.org/10.1080/1539152 3.2001.10782344

Brown, J. \& Duguid, P. (1993). Stolen knowledge. Educational Technology. March, 10-15, New Jersey, USA: Educational Technology Publications.

Chaouchi, H. (2010). The Internet of things: Connecting objects. John Wiley and Sons, Inc. (Eds.). USA. 265 p.

Daniel, J. (2012). ICTs in global learning/teaching/training. UNESCO Institute for information technologies in education. Policy brief. UNESDOC-UNESCO (Eds.). Moscow, Rusia. 12 p. http://unesdoc.unesco.org/images/0022/002202/220239E.pdf.

Darwin, C. (2010). El origen de las especies. EDAF (Eds.). Madrid, España. 696 p.

David K. \& Ben, Y. (2012). Using ICT for school purposes: Is there a student-school disconnect? Computers \& Education, 59(3), 907-914. http://doi.org/10.1016/j.compedu.2012.04.012
Denavit, J. \& Hartenberg, R.S. (1965). A kinematic notation for lower-pair mechanisms based on matrices, Transactions ASME. J Appl Mech, 22(2), 215 - 221.

Dobzhansky T., Ayala F.J., Stebbins G.L. \& Valentine J.W. (1993). Evolución. Omega (Eds.). Barcelona, España. 239 p.

Garnica, E. (2013). Robots Herramientas para las Aulas de Clase. Rev Ingeniería, Matemáticas y Ciencias de la Información, 1(1), 31-43. http:// urepublicana.edu.co/ingenieria/wp-content/ uploads/2014/04/Robots.pdf.

Hatch, T. \& Seidel, S. (1997). Putting the student work in the table. The Phi Kappa Phi Journal, 77(1), 18-21.

Haythornthwaite, C. (2005). Social networ$\mathrm{ks}$ and Internet connectivity effects. Inform Commun Soc, 8(2), 125-147. http://dx.doi. org/10.1080/13691180500146185

Jiménez, L. (2013). Enseñanza de la tecnología basada en cursos de robótica práctica: proyectos educativos con lego y con dispositivos propios. Tesis doctoral en Educación. Universidad Santo Tomás. Bogotá, Colombia.

Lancheros, D. \& Rubiano, A. (2009). Diseño de software en módulo didáctico de aprendizaje para la construcción, implementación y manipulación de Robots. Caso de estudio: "Robot tipo Topo". Informe de investigación en proceso. Ingeniería Solidaria, 5 (9), 8-13.

Lancheros, D. (2010). Diseño e implementación de un módulo didáctico para el aprendizaje en la construcción, implementación y manipulación de robots. Formación Universitaria, 3 (5), 3-8

Margolis M. (2011). Arduino Cookbook. 2nd Edition. O'Reilly (Eds.). USA. 724 p. ftp://facfiet.unicauca. edu.co/TalleresIoT/Libros/Arduino-Cookbook-Michael.Margolis.pdf.

Márquez D., Jairo E., Ruiz F. \& Javier H. (2014). Robótica Educativa aplicada a la enseñanza básica secundaria. Didáctica, Innovación y Multimedia (DIM), 10 (30) ,1-12. http://www.raco.cat/index. php/DIM/article/view/291518/379999.

Patiño, K., Belén, C. \& Vidal, R. (2010). Experiencias construccionistas con robótica educativa en el centro internacional de tecnologías avanzadas. Buenas prácticas de enseñanza con TIC. Revista Electrónica Teoría de la Educación: Educación y Cultura en la Sociedad de la Infor- 
mación, 11(1), 310-329. http://www.redalyc.org/ pdf/2010/201014897013.pdf.

Seidel, S. \& Walters, J. (1994). The things children make in school: Disposabel or indispensable? Harvard Graduate School of Education Alumni Bulletin, 39 (1), 8-20.

Simon, M. (2011). Programming Arduino: getting started with sketches. McGraw-Hill (Eds.). USA. 176 p.

Valenzuela, B. \& Pérez, M. (2013). Aprendizaje autorregulado a través de la plataforma virtual Moodle. Educ 16 (1), 66-79.

Watson, D. (2001). Pedagogy before technology: Re-thinking the relationship between
ICT and teaching. Education and Information Technologies, 6 (4), 251-266. http://dx.doi.org/10.1023/A:1012976702296

Webster. (2009). The Merriam-Webster dictionary. Merriam-Webster Inc. (Eds.). Springfield, USA. $967 p$.

Yang, S. (2008). A comprehensive study of the regulation and behavior of web crawlers. Doctoral tesis. Pennsylvania State University. Pennsylvania, USA. 345 p. 